\title{
Methodological Aspects of Integrative and Person- Oriented Health Care Evaluation
}

\author{
Gunver Sophia Kienle ${ }^{a, b, c}$ Harald Johan Hamre ${ }^{a, b}$ Helmut Kiene Ha,b $^{a}$ Thomas Ostermann ${ }^{b, d}$ \\ Laura Anderle $^{e}$ Nadine Maussner $^{d}$ Reinhard Schuster ${ }^{f}$
}

a Institute for Applied Epistemology and Medical Methodology at Witten/Herdecke University, Freiburg, Germany;

${ }^{\mathrm{b}}$ Institute for Integrative Medicine, Faculty of Health, Witten/Herdecke University, Herdecke, Germany;

${ }^{\mathrm{c}}$ Center for Complementary Medicine, Institute for Environmental Health Sciences and Hospital Infection Control, Medical Center, University of Freiburg, Freiburg, Germany;

${ }^{\mathrm{d} D e p a r t m e n t}$ of Psychology and Psychotherapy, Witten/Herdecke University, Witten, Germany;

e Faculty of Management and Economics, Witten/Herdecke University, Witten, Germany;

${ }^{\mathrm{f}}$ Institute of Mathematics, University of Lübeck, Lübeck, Germany

\section{Integrative Evaluation of Whole Medical Systems and Person-Centered Care}

\section{Authors: Gunver Sophia Kienle, Harald Johan Hamre, Helmut Kiene}

Whole Medical Systems (WMSs) are complete systems of medical theory and practice; they assume the human being as a hierarchically structured and interrelated system; they see diseases within this holistic paradigm, affecting and being affected by different parts of the human individual, a disease or condition thus having general and unique aspects in each patient; besides the physical and (patho-)physiological level, also the emotional, biographical, social, and spiritual dimensions are regarded to be important. This introduces a person-centered, individualized approach.

Many complementary therapy systems are WMSs (e.g., Anthroposophic Medicine, Ayurveda, Homeopathy, Naturopathy, Traditional Chinese Medicine). They have distinct nosological conceptions, diagnostic procedures, and integrate sometimes thousands of treatment approaches, e.g. remedies, non-pharmacological interventions, lifestyle recommendations, counseling, and support the patient pursuing his or her own goal. The treatments are mostly deduced from the WMSs concept or derived from its medical practice, but rarely from molecular reasoning or preclinical experiments. Indications for remedies from WMSs are not restricted to singular disease entities; one remedy is often applied in a large variety of diseases, depending on the individual condition and situation of the patient (see below the contribution by Hamre et al. in this article). In general, these complementary therapy systems are integrated with conventional medicine, representing a form of integrative and personalized health care [1].
WMS therapies often are multimodal in order to address both the typical as well as the individual presentation of the disease, to address different conditions of the patient presumed to be connected with the disease, and also to strengthen his or her salutogenetic potentials. Thus, multimodal interventions often intend to support each other's working principle. Frequently, the treatments are delivered by cooperating providers (physicians, therapists, nurses, nutritionist, etc.), the delivery depending on characteristics and competence of the provider and the patient. Many of the interventions are to be flexibly and individually tailored.

Due to these characteristics, WMSs present challenges for the scientific and medical comprehension and evaluation. The traditional research model ( 1 trial for 1 intervention in 1 disease) does not suffice due to a variety of reasons, particularly as it does not account for the system synergies and patient centeredness. It would also produce an unsurmountable number of trials (e.g., 1 WMS with 1,000 remedies, 50 potential indications each, would require 50,000 trials, necessitating millions of study patients, billions of Euros, without addressing multimodal and individualized application). Research strategies have been proposed to adequately evaluate WMSs and address their complexity, the system aspects, and the patient centeredness [2].

Whole-system research assumes that in a system the whole is more than the parts, producing emergent effectiveness. It is crucial to study the intact, whole, and complex system, including the synergies of multimodal treatments, the system context, the patientprovider relationship, and the treatment individualizations (system research is equally pursued for complex interventions in conventional medicine, such as stroke care or diabetes care). In further steps, exemplary individual treatments are to be investigated in more detail and depth, using modern clinical and preclinical re- 
search methodologies and equipment. Other important evaluation issues are the professionalism of the care provision, ethical issues, treatment safety, basic research, and to get insight into the modes of care individualization and of patient perspective. Also, its relationship to conventional medical treatment, like its added value, comparative effectiveness, or interaction, should be analyzed.

To achieve an integrative evaluation, the following aspects of the WMSs should be assessed [2]:

1) Therapeutic professionalism and ethical issues: definition, teaching, evaluation.

2) The patient perspective as well as public need and demand regarding the availability of the specific medical system within the general health care system.

3) The conceptual basis, as presented in preclinical research, like physiological, biochemical, cellular research analyzing the underlying principles of action, and as presented in epistemological research on the WMS concepts and working principles, including its conception of man and nature, and how these concepts relate to those of other therapy systems; also, diagnostic procedures relating to distinct nosological conceptions should be assessed.

4) Efficacy, effectiveness:

- Assessment of the medical system as a whole, with consideration of its specific diagnostic and therapeutic procedures; these may be large cohort studies, pragmatic randomized controlled trials (RCTs) or non-randomized comparative studies, or else (e.g., Hamre et al. below in this article).

- Exemplary interventions should be investigated in highquality, well-designed large RCTs or non-randomized comparative trials; the treatments to be investigated are those significant for the WMS, those that have a strong indication in one disease and relevance also outside the WMS for a relevant health condition, and for which some empirical data on its effects in the clinical condition and on its safety are available (there may be reservation against randomized treatment allocation within the WMS, particularly when the treatments are regarded to be very effective and the condition is a serious burden for the patient; therefore, the trials may have to be conducted outside the WMS).

- The bulk of remaining therapies are to be investigated or documented with smaller studies or RCTs, case series, case reports, specific analyses of subgroups in large system observation studies, matched-pair studies, and synthesis from other evidence on the same type of intervention.

- The quality and results of these studies have to be assessed in systematic reviews or meta-analyses; review methods for whole-system research and case reports still need to be developed.

5) Safety is of utmost importance and is assessed with a variety of methods: e.g. in efficacy and effectiveness studies, in pharmacoepidemiological studies, in case reports, in specific assessments of certain questions, by pharmaceutical quality control, and by evaluation of professional application.
6) Costs can be assessed with a variety of objectives, methods, and perspectives.

7) Assessment of individualized, personalized therapies and their outcomes, and insight into individual decision-making, treatment procedures, clinical judgment, and observations of the clinician's work can be achieved by qualitative methods, ethnographic studies, and high-quality case reports or case studies. These individualized assessments can also be combined with quantitative studies for a mutual increase of quality and depth of information.

Integrative Evaluations of WMSs cover the above-mentioned dimensions. They integrate evidence from different sources, based on different methods, each of them with specific types of internal, external, and construct validity, and specific quality criteria. The assessment methods are largely available and can be referred to internationally acknowledged standards. This is particularly true for clinical trials and observational studies. Still, some of the methods have to be adapted, refined, or expanded for the specific purposes of WMS research. In particular, whole-system studies still are pioneer work, and so is single case research.

Likewise important for the integrative evaluation of WMSs and person-centered care is the integration of expertise and perception of the relevant health care stakeholders into the research process: patients, health care providers from the WMS as well as conventional medicine, teachers, methodologists, funders, insurances, the industry, guideline developers, politicians, and health authorities.

Integrative evaluation means to integrate the results from the different sorts of research and organize them in such a way that they present an overall and differentiated picture of the WMS, depending on the reader's or user's interest. This provides transparency of the WMS in all relevant fields to the general health care system and its relevant stakeholders, to the public, policy makers, and scientists.

\section{Acknowledgement}

All authors are supported by the Software AG Stiftung, Darmstadt. The Stiftung has no influence on the content of their research projects and publications.

\section{Disclosure Statement}

All authors declare that there is no conflict of interest concerning this paper.

\section{References}

1 Heusser P, Neugebauer E, Berger B, Hahn EG: Integrative und personalisierte Gesundheitsversorgung - Forderungen für ein zeitgemäßes Gesundheitssystem. Gesundheitswesen 2012;75:151-154.

2 Kienle GS, Albonico H-U, Fischer L, et al: Complementary therapy systems and their integrative evaluation. Explore (NY) 2011;7:175-187. 


\section{Modeling Patient-Practitioner Interaction by Means of a Dynamical System}

\section{Authors: Thomas Ostermann, Laura Anderle, Nadine Maussner, Reinhard Schuster}

Background: Person-centered medicine focuses 'on the role and worth of the person in medicine, the cruciality of sense of identity, empathy and engagement for optimal clinical care' [1]. Thus, the interaction of patient and practitioner is one of the key elements of person-centered medicine, which has been outlined in a variety of studies from all fields of patient care [2, 3]. In particular, studies have revealed that the practitioners' capacity to attend to their patients may have an impact on the therapeutic outcome [4]. Moreover the 'interaction style' [5] might influence the therapeutic outcome and may lead to a significant symptom improvement [6]. The Patient-practitioner interaction thus can be seen as a moderator component in the therapeutic context, influencing 'health outcomes across diverse interventions and conditions' [7]. But although the dynamics of the patient-practitioner interaction are seen as an important element in patient-centered medicine, an approach to model such effects mathematically has not been introduced in the field of person-centered medicine so far.

Methodological approach: One mathematical approach we have identified originates from the field of modeling interpersonal relationships and has been introduced by Strogatz in 1988 [8] and rediscovered by Rinaldi [9] and Sprott [10, 11].

For 2 individuals (mostly denoted R for Romeo and J for Juliet), their relationship is given by 2 equations:

$$
\begin{aligned}
& \frac{d R}{d t}=a R+b J+f \\
& \frac{d J}{d t}=c J+d R+g
\end{aligned} \quad \text { with } a, b, \ldots, f, g \in I R
$$

Each equation describes the change in relationship of both individuals, e.g. in Juliet, $\mathrm{d} J / \mathrm{dt}$, depending on the state of Romeo $(\mathrm{dR})$, the state of Juliet (cJ), and an initial state (g). These 2 differential equations depend on each other and thus form a so-called dynamical system. Hence, the parameters a to d denote the interaction style or, according to Gottman et al. [12], the 'behavioral inertia' and 'influence function'. The parameters $\mathrm{f}$ and $\mathrm{g}$ represent the appeal (if $>0$ ) or repulsion (if $<0$ ) [13]. The case of $f=g=0$ corresponds to a relationship with neither initial appeal nor repulsion [11]. This model has been extended by the replacement of the linear terms $\mathrm{bJ}$ and $\mathrm{dR}$ with the nonlinear logistic terms bJ(1J) and $d R(1-R)$, respectively [10]. This extension allows the modeling of nonlinear interactions. Finally, Ghosh [14] proposed a third equation introducing the effort $\mathrm{E}$ of Romeo in order to 'attract Juliet and to generate / increase feeling of love in her mind for him':

$$
\begin{gathered}
\frac{d R}{d t}=a R+b J(n-J)+p \\
\frac{d J}{d t}=c R(m-R)+d J+c E(u-E)+q \\
\frac{d E}{d t}=f R+g J(n-J)+r
\end{gathered}
$$

Hence, the term cE(u-E) only acts on Julia (second line) and $\mathrm{E}$ is not static but depends linearly on Romeo and nonlinearly on Juliet. The letters $\mathrm{n}$ and $\mathrm{m}$ denote the individual critical levels of a positive response, and $\mathrm{u}$ denotes the critical level of Romeo's engagement.

In 2011, Liebovitch et al. [15] proposed to use a dynamical system similar to equation 1 in a therapeutic context and to model the success of psychotherapy by replacing Romeo with the therapist $\mathrm{T}$ and Julia by the client $\mathrm{C}$. $\mathrm{T}$ and $\mathrm{C}$ can thus be seen as the subjective state and feeling that the therapist and the client show to each other. Considering the extended model of Ghosh, we now would propose to use the effort function $\mathrm{E}$ which now represents the therapeutic engagement of the therapist.

Figures $1 \mathrm{a}$ and $\mathrm{b}$ exemplify the trajectories of the system dynamics for 2 situations. In situation 1, the therapist's input in the relationship is higher than the input he gets from the client ( $\mathrm{a}=$ $0.532 ; b=0.2257)$. The same holds for the client. His own input in the relationship is also half as much as the one from the therapist ( $c=0.43753 ; d=0.85792)$, with a similar coefficient for the effort of the therapist ( $e=0.83485$ ) influencing the relationship between them. The effort function itself is influenced with almost the same magnitude from the therapist and the client ( $\mathrm{f}=0.9602$; $\mathrm{g}=$ $0.9294)$. Finally, all equations start with more or less the same constant values $(\mathrm{p}=0.9989 ; \mathrm{q}=1.005 ; \mathrm{r}=0.9968)$. For simplicity, $\mathrm{n}, \mathrm{m}$, and $\mathrm{u}$ are set equal to 1 .

In situation 2, we have almost the same parameters ( $\mathrm{a}=$ $0.531442 ; \mathrm{b}=0.2302 ; \mathrm{c}=0.43793 ; \mathrm{d}=0.85778 ; \mathrm{e}=0.8304 ; \mathrm{f}=$ $0.9346 ; \mathrm{g}=0.9486 ; \mathrm{p}=0.99619 ; \mathrm{q}=0.97674 ; \mathrm{r}=0.99055 ; \mathrm{n}=\mathrm{m}=$ $\mathrm{u}=1$ ) which, however, show a qualitatively different solution (fig. 1b). In both cases, after a period of time, the system collapses.

It has to be noted that all parameters in the model were chosen to demonstrate how the system works in principle. This also shows that slight differences in these parameters may have a huge impact on the dynamics of the system. A closer look inside the mathematical structure of the system also reveals that states of equilibrium do exist in principle. This can be seen in the trajectories in figures 1a and $\mathrm{b}$ which start in a balanced situation, but show diverging developments after a certain amount of time. Thus, an initially 'balanced relationship' between client and therapist becomes instable in this model.

Discussion: The interaction of client and therapist is one of the key elements of person-centered medicine. We aimed at providing a mathematical framework to model such interaction. We were able to present some solutions which, however, failed to show a convergent and stable behavior over the course of time. Although in principle it is not excluded to find a longer interval with a stable 


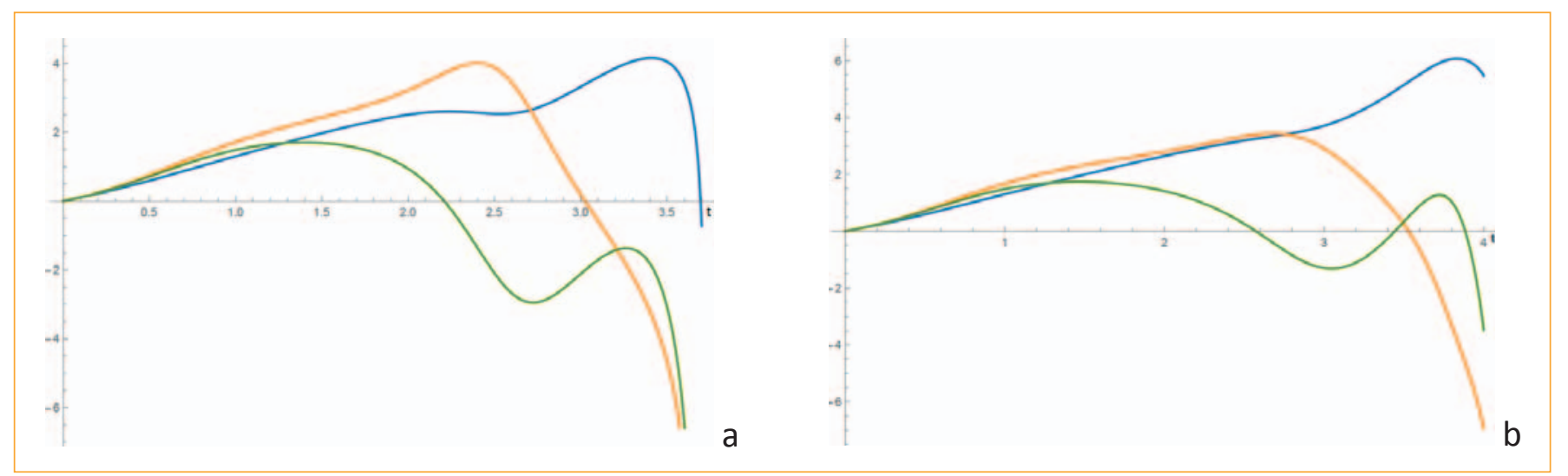

Fig. 1. a and $\mathbf{b}$ : trajectories of the dynamical model (blue line: therapist; yellow line: client; green line: effort function; the $x$-axis represents the time (t)).

solution, and despite the fact that we did not mathematically prove the existence of stable solutions of the dynamical system, we were able to show that it reacts quite sensible to small changes in its parameters as presented in figures $1 \mathrm{a}$ and $\mathrm{b}$. Thus, the practical application of the current system might be limited. Therefore, we suggest modifying this modeling approach with a higher damping term which in a first approximation can be interpreted as a faster response in the therapist-client interaction.

From a practical point of view, all of these models are currently only of theoretical nature and thus require empirical investigation to test their validity. One approach to investigate the validity of the models might be given in using the work alliance inventories [16, 17].

\section{Disclosure Statement}

None of the authors has any conflict of interest to declare regarding this article.

\section{References}

1 Mezzich JE, Snaedal J, Van Weel C, et al: Introduction to person-centred medicine: from concepts to practice. J Eval Clin Pract 2011;17:330-332.

2 Escher M, Büssing A, Ostermann T: Survey on hand gestures relevance in patient practitioner communication: a homeopathic example. Homeopathy 2016;105:233-239.

3 Beitat K: Trust and Incidents: The Dynamic of Interpersonal Trust between Patients and Practitioners. Wiesbaden, Springer, 2015.

4 Crepeau EB: 'I need someone to keep an eye on me': the power of attention in patient-practitioner interactions. Disabil Rehabil 2016;38:2419-2427.

5 Vinall-Collier K, Madill A, Firth J: A multi-centre study of interactional style in nurse specialist- and physician-led Rheumatology clinics in the UK. Int J Nurs Stud 2016;59:41-50.

6 Holmes RD, Tiwari AK, Kennedy JL: Mechanisms of the placebo effect in pain and psychiatric disorders. Pharmacogenomics J 2016;16:491-500.

7 Bishop FL, Lewith GT: A qualitative exploration of how patients conceptualise their acupuncturists: technicians, caring professionals, and wise healers. Complement Ther Med 2016;27:74-81.

8 Strogatz SH: Love affairs and differential equations. Math Magazine 1988; 61:35.
9 Rinaldi S: Love dynamics: the case of linear couples. Appl Math Comp 1998; 95:181-192.

10 Sprott J: Dynamical models of love. Nonlinear Dyn Psych Life Sci 2004;8: 303-314.

11 Sprott JC: Dynamical models of happiness. Nonlinear Dyn Psych Life Sci 2005;9:23-36.

12 Gottman JM, Murray JD, Swanson CC, et al: The mathematics of marriage. Cambridge, MA, MIT Press, 2002.

13 Wauer J, Schwarzer D, Cai GQ, Lin YK: Dynamical models of love with time-varying fluctuations. Appl Math Comput 2007;188:1535-1548.

14 Ghosh K: Love between Two Individuals in a Romantic Relationship: A Newly Proposed Mathematical Model. The 7th IMT-GT International Conference on Mathematics, Statistics and its Applications 2011, pp 978-974.

15 Liebovitch LS, Peluso PR, Norman MD, et al: Mathematical model of the dynamics of psychotherapy. Cogn Neurodyn 2011;5:265-275.

16 Petrowski K, Nowacki K, Pokorny D, Buchheim A: Matching the patient to the therapist, the roles of the attachment status and the helping alliance. J Nerv Ment Dis 2011;199:839-844.

17 Flückiger PDC, Horvath AO, Del Re AC, et al: Bedeutung der Arbeitsallianz in der Psychotherapie. Psychotherapeut 2015;60:187-192.

\section{System Evaluation Studies of Integrative and Personalised Health Care - Examples from Anthroposophic Outpatient Treatment}

\section{Authors: Harald Johan Hamre, Helmut Kiene, Gunver Sophia Kienle}

Anthroposophic medicine (AM) is a system of integrative and personalised health care (IPHC) [1]: provided by physicians and non-medical therapists, AM integrates specific AM therapy modalities (special medications; counselling; special art, movement and physical therapies) with conventional treatment $(\mathrm{CON})$ and sometimes other, non-AM complementary or alternative treatments [2]. AM focuses on the whole person, addressing physical, biological, emotional, biographical and spiritual levels in health and disease. Furthermore, AM therapy is often individualised or personalised: the AM conception of man includes a number of ty- 
pological categorisations relevant for diagnostics and therapy, such as 2 (upper and lower) poles, 3 dynamic systems, 4 levels of formative forces and 7 planetary or metal qualities [3]. Accordingly, different patients with the same diagnosis according to conventional medicine may have different typological profiles, each requiring a different, i.e. an individualised AM treatment. Finally, the broad outlook and individualisation of treatment also affect the practitioner-patient relationship. For example, physicians providing AM often have prolonged consultations with their patients in order to take a detailed case history with respect to typology profiling and to address biographic and existential/spiritual aspects of the patients' illness.

For clinical research, certain features of IPHC need to be attended to. This will be illustrated by 3 prospective studies on IPHC (AM $+\mathrm{CON}$ as needed) in outpatient settings:

- AMOS (Anthroposophic Medicine Outcomes Study): a 4-year observational study on patients with various chronic diseases starting AM treatment: eurythmy, art or rhythmical massage therapy; medical treatment (prolonged consultations with AM physicians followed by new prescription of AM medication). AMOS was a single-arm study with a nested non-randomised comparative study on IPHC (AM + CON as needed) vs. CON for low back pain [4].

- IIPCOS-Anthroposophic Medicine (International Integrative Primary Care Outcomes Study): a 4-week comparative observational study on patients starting treatment for acute respiratory or ear infections, seeing physicians offering IPHC (AM + CON as needed) or CON alone [5].

- CPAK (German acronym for 'anthroposophic vs. conventional treatment for early rheumatoid arthritis'): a 4-year non-randomised controlled trial on patients with early rheumatoid arthritis starting treatment in outpatient clinics offering IPHC (AM + CON as needed) or CON alone (Hamre et al., submitted for publication; Universal Trial Number U1111-1187-5015).

As per definition, IPHC is multimodal and complex [1], whereby the number and combination of therapy modalities and components (e.g., different AM medications) may differ across settings. In order for the results of IPHC treatment studies to be generalisable, and for comparison to other studies, it is essential to define, document and report relevant aspects of IPHC treatment, including the providers, components and delivery of therapy. For studies in which IPHC consists of an add-on to a reference treatment (e.g., $\mathrm{AM}+\mathrm{CON}$ ), it is particularly important to define and document the use of the reference treatment in question.

- For each of the 3 AM studies, eligibility criteria for the participation of clinics (in CPAK), physicians and (in AMOS) nonmedical therapists were pre-defined. In AMOS and IIPCOS, AM therapy providers were certified by the respective national physician or therapist association. In IIPCOS, an additional eligibility criteria for participating AM physicians was the regular prescription of AM medications to $\geq 75 \%$ of their patients with upper respiratory and ear infections in order to maximise the contrast between the IPHC $(\mathrm{AM}+\mathrm{CON})$ and CON study arms [5].
- In all 3 studies, operational criteria for AM medications and non-medication AM therapy modalities were defined. In AMOS, also the date of each session with eurythmy, art or rhythmical massage therapy was documented [6]. In AMOS and IIPCOS, the duration of the initial physician consultation was documented, in AMOS also the duration of prolonged follow-up consultations [7].

- AMOS comprised patients with different chronic diseases. For the most frequent indications, guideline-supported conventional treatments were identified and their use documented $[4,8]$. In IIPCOS, the use of antibiotics and analgesics was of special interest $[5,9]$. In CPAK, corticosteroids and non-steroid antiinflammatory drugs were permitted in both study arms, while disease-modifying anti-inflammatory drugs were prohibited in the IPHC $(\mathrm{AM}+\mathrm{CON})$ arm and mandatory in the CON arm. Initial evaluation of IPHC therapy will often focus on effects of the whole IPHC intervention package [10]. However, it may also be desirable to assess the impact of specific therapy components as well as other factors, apart from the interventions. In AMOS, subgroup analyses were conducted according to the primary AM treatment component for each patient (eurythmy, art, rhythmical massage, medical). In AMOS [11] and IIPCOS [9], factors associated with the primary clinical outcomes were identified by predictor analyses. And in AMOS, combined effects of 5 factors apart from the AM treatment (selection bias, non-respondent bias, natural recovery, regression to the mean, use of standard conventional treatments) were analysed by a combination of sample restriction and simple adjustment procedures $[4,8]$.

For the assessment of adverse effects in IPHC studies, it is important to know which therapy component they were caused by. In all 3 AM studies, suspected adverse reactions to any treatment were documented. In addition, all suspected reactions to medications (in AMOS: AM medications only [12]) were assessed individually for causality on the patient level [13].

This overview illustrates some methodological issues pertaining to clinical studies on IPHC. Research strategies towards IPHC should also address the role of the CON component therein and its possible modification by the other components.

\section{Acknowledgements}

This paper was funded by the Software AG Stiftung. The sponsor had no influence on the preparation, review or approval of the manuscript.

\section{Disclosure Statement}

In the past 3 years, the authors received research grants from the European Coalition of Homeopathic and Anthroposophic Medicinal Products, Wala Heilmittel GmbH and Weleda AG and honorariums and/or travel expense reimbursements from Abnoba GmbH, Helixor Heilmittel GmbH, Iscador AG and Weleda AG. 


\section{References}

1 Heusser P, Neugebauer E, Berger B, Hahn EG: Integrative und personalisierte Gesundheitsversorgung - Forderungen für ein zeitgemäßes Gesundheitssystem (Integrative and personalized health care - requirements for a timely health-care system). Gesundheitswesen 2013;75:151-154.

2 Kienle GS, Albonico HU, Baars E, et al: Anthroposophic medicine: an integrative medical system originating in Europe. Glob Adv Health Med 2013;2: $20-31$.

3 Girke M: Internal Medicine: Foundations and Therapeutic Concepts of Anthroposophic Medicine. Berlin, Salumed-Verlag, 2012.

4 Hamre HJ, Kiene H, Ziegler R, et al: Overview of the publications from the Anthroposophic Medicine Outcomes Study (AMOS) - a whole system evaluation study. Global Adv Health Med 2014;3:54-70.

5 Hamre HJ, Fischer M, Heger M, et al: Anthroposophic vs. conventional therapy of acute respiratory and ear infections: a prospective outcomes study. Wien Klin Wochenschr 2005;117:256-268.

6 Hamre HJ, Kiene H, Glockmann A, et al: Long-term outcomes of anthroposophic treatment for chronic disease: a four-year follow-up analysis of 1510 patients from a prospective observational study in routine outpatient settings. BMC Res Notes 2013;6:269.
7 Hamre HJ, Witt CM, Glockmann A, et al: Anthroposophic medical therapy in chronic disease: a four-year prospective cohort study. BMC Complement Altern Med 2007;7:10.

8 Hamre HJ, Glockmann A, Kienle GS, Kiene H: Combined bias suppression in single-arm therapy studies. J Eval Clin Pract 2008;14:923-929.

9 Hamre HJ, Glockmann A, Schwarz R, et al: Antibiotic use in children with acute respiratory or ear infections: prospective observational comparison of anthroposophic and conventional treatment under routine primary care conditions. Evid Based Complement Alternat Med 2014;2014:243801.

10 Fønnebø V, Grimsgaard S, Walach H, et al: Researching complementary and alternative treatments - the gatekeepers are not at home. BMC Med Res Methodol 2007;7:7.

11 Hamre HJ, Witt CM, Kienle GS, et al: Predictors of outcome after 6 and 12 months following anthroposophic therapy for adult outpatients with chronic disease: a secondary analysis from a prospective observational study. BMC Res Notes 2010;3:218.

12 Hamre HJ, Witt CM, Glockmann A, et al: Use and safety of anthroposophic medications in chronic disease: a 2 -year prospective analysis. Drug Saf 2006; 29:1173-1189.

13 Hamre HJ, Glockmann A, Fischer M, et al: Use and safety of anthroposophic medications for acute respiratory and ear infections: a prospective cohort study. Drug Target Insights 2007;2:209-219. 